\title{
Intelligent Parking System Using IOT
}

\author{
Sushma, Sushma M*, Rachana Raj A N, Zoya Fathima, Shruthi B M \\ Department of CSE, GSSSIETW, Mysuru, Karnataka, India
}

DOI: https://doi.org/10.21467/proceedings.1.29

* Corresponding author email: sushmamallesh154@gmail.com

\begin{abstract}
In recent times the concept of smart cities has gained grate popularity. Thanks to the evolution of Internet of things the idea of smart city now seems to be achievable. Consistent efforts are being made in the field of IoT in order to maximize the productivity and reliability of urban infrastructure. Problems such as, traffic congestion, limited car parking facilities and road safety are being addressed by IoT. In this paper, we present an IoT based cloud integrated smart parking system. The proposed Smart Parking system consists of an on-site deployment of an IoT module that is used to monitor and signalize the state of availability of each single parking space. A mobile application is also provided that allows an end user to check the availability of parking space and book a parking slot accordingly. The paper also describes a high-level view of the system architecture. Towards the end, the paper discusses the working of the system in form of a use case that proves the correctness of the proposed model.
\end{abstract}

Index Terms-Internet of things, Cloud computing, Automobiles, Mobile applications, Smart cities, Servers;

\section{INTRODUCTION}

The concept of Internet of Things (IoT) started with things with identity communication devices. The devices could be tracked, controlled or monitored using remote computers connected through Internet. IoT extends the use of Internet providing the communication, and thus inter-network of the devices and physical objects, or 'Things'. The two prominent words in IoT are "internet" and "things". Internet means a vast global network of connected servers, computers, tablets and mobiles using the internationally used protocols and connecting systems. Internet enables sending, receiving, or communicating of information. Thing in English has number of uses and meanings. Dictionary meaning of 'Thing' is a term used to reference to a physical object, an action or idea, situation or activity, in case when we do not wish to be precise. IoT, in general consists of inter-network of the devices and physical objects, number of objects can gather the data at remote locations and communicate to units managing, acquiring, organizing and analyzing the data in the processes and services. It provides a vision where things (wearable, watch, alarm clock, home devices, surrounding objects with) become smart and behave alive through sensing, computing and communicating

(C) 2018 Copyright held by the author(s). Published by AIJR Publisher in Proceedings of the $3^{\text {rd }}$ National Conference on Image Processing, Computing, Communication, Networking and Data Analytics (NCICCNDA 2018), April 28, 2018.

This is an open access article under Creative Commons Attribution-NonCommercial 4.0 International (CC BY-NC 4.0) license, which permits any non-commercial use, distribution, adaptation, and reproduction in any medium, as long as the original work is properly cited. ISBN: 978-81-936820-0-5 
by embedded small devices which interact with remote objects or persons through connectivity. The scalable and robust nature of Cloud computing is allowing developers to create and host their applications on it. Cloud acts as a perfect partner for IoT as it acts as a platform where all the sensor data can be stored and accessed from remote locations [11]. These factors gave rise to the amalgamation of bothtechnologies thus leading to the formation of a new technology called Cloud of Things(CoT). In CoT the things(nodes) could be accessed, monitored and controlled from any remote location through the cloud. Due to high scalability in cloud any number of node could be added or removed from the IoT system on a real time basis. In simple terms IoT can be explained in form of an equation stating:

\section{LITERATURE SURVEY}

There are several methods employed for the vehicle parking. The concept of new smart parking solves the parking problem by using mixed integer linear problem. The disabled person can park the vehicle at specially designed locations.

\subsection{Optimal resource allocation and reservation}

YanfengGeng and Christos G. Cassandras proposed the concept of "A new smart parking system based on optimal resource allocation and reservations". Drivers access the system via cellular phone or internet. a new concept for a "smart parking" system. This system explicitly allocates and reserves optimal parking spaces to drivers. It uses the concept of mixed integer linear problem. Drivers who are looking for parking spots send requests to the DPRC. Driver Processing Request Centre gathers driver parking requests Cars location keeps track of driver allocation status and sends back the assignment result to drivers. A request is based on parking costs and walking distance between a parking spot and the driver's actual destination. It also contains the driver's basic information such as license number, current location and car size. An assigned parking space is send back to each driver via the DPRC. If the driver is satisfied with the assignment he has the choice to reserve that spot. Once reservation is made the driver still has opportunities to obtain a better spot. The PMRC [Parking Resource Management Center] then updates the corresponding parking spot from vacant to reserve and ensures that other drivers have no permission to take that spot. Parking Resource Management Centre collects and updates all real time information and disseminates it via internet. If a driver is not satisfied with the assignment or he fails to accept it for any other reason he has to wait until the next decision point. The mixed integer linear problem solves problems at each decision point.

\subsection{Disassist: Parking for disabled person}

"Disassist: An internet of things and mobile communications platform for disabled parking space management" proposed by Lambros Lambrinos and Aristotle's Dosis. It is a system designed and developed based on the principles brought forward by IOT and smart cities

Proceedings of the $3^{\text {rd }}$ National Conference on Image Processing, Computing, Communication, Networking and Data Analytics (NCICCNDA 2018) 
Intelligent Parking System Using IOT

initiatives. It integrates sensors and Smartphone's along with wireless and mobile communications to provide for better utilization and management of parking spaces allocated for use by people with disabilities. When we go through a city during rush hours when traffic is at its peak, all parking spaces are already occupied. A person with disability is trying to access city centre but he did not find any disabled parking slot. There are drivers who occupy disabled parking slots without having the right to do so. Even in some cases there are violators who have fake documents displayed on their window screen. The aim of the disassist is to enhance the parking experience from the perspective of people with disabilities. In this system the parking management organized as parking bays. The parking bays can be on street and off street. In on street, the Parking bays are found on the roadside. In off street, different categories of parking loads are ranging from single areas to large multi-storey car parks. Monitoring parking spaces are not only for checking fee payments but also for calculating availability and reporting such information to drivers for searching vacant spaces. Parking reservation can be implemented relatively easily in controlled areas that can theoretically enforce reservations and hence guarantee the availability of the reserved space. In open spaces actions such as user verification and reservations are more difficult to accomplish. When a reservation remains valid and charging must start when vehicle actually arrives at the parking location. The parking space locations are displayed on a map allowing drivers to navigate to these locations. The level of information is related to its vehicle capacity and hourly fees. A real advantage is provided by those that report current availability and reservations. A significant number of people suffer from temporary or permanent disabilities. A special permit called a disabled badge is to be issued to these people.

\subsection{Using Android Application}

"Automated car parking system commanded by android application" introduced by D.J. Bonde. The android application generates automatic parking and un parking with the help of commands of an android application. The system reduces the International Journal of Engineering Science and Computing, November 20163177 http://ijesc.org/ human intervention to the minimum by automating the process of car parking. When we visit various public spaces like shopping malls, five stars/seven star hotels, multiplex cine halls many problems relating to the availability of parking spaces. Most of the times we need to traverse through multiple parking slots to find a free space for parking. Our proposed system presents an autonomous car parking that regulates the number of cars that can be parked in a given space at any given time based on the parking space availability. When a car arrives at the entrance, it will be stopped at the main gate and the driver beboards the car. Using the android application on his android device, the user commands the Parking Control Unit to check the status of available parking slots, through an SMS. On receiving this command, a search for free slot is carried out and corresponding information is provided to the user by means of SMS. If the availability of parking space is confirmed, the user commands the car to the designated slot. The car traces its path to the entrance of the parking area. The proposed 
architecture consists of a car control unit and parking control unit. It has four modules, first is interfacing LCD with atmega32 microcontroller. It is used for displaying the status of parking. Depending on the status LCD counter is incremented or decremented. Second is, interfacing GSM Sim 900 with atmega 32 microcontroller. GSM means global systems for mobile communication.

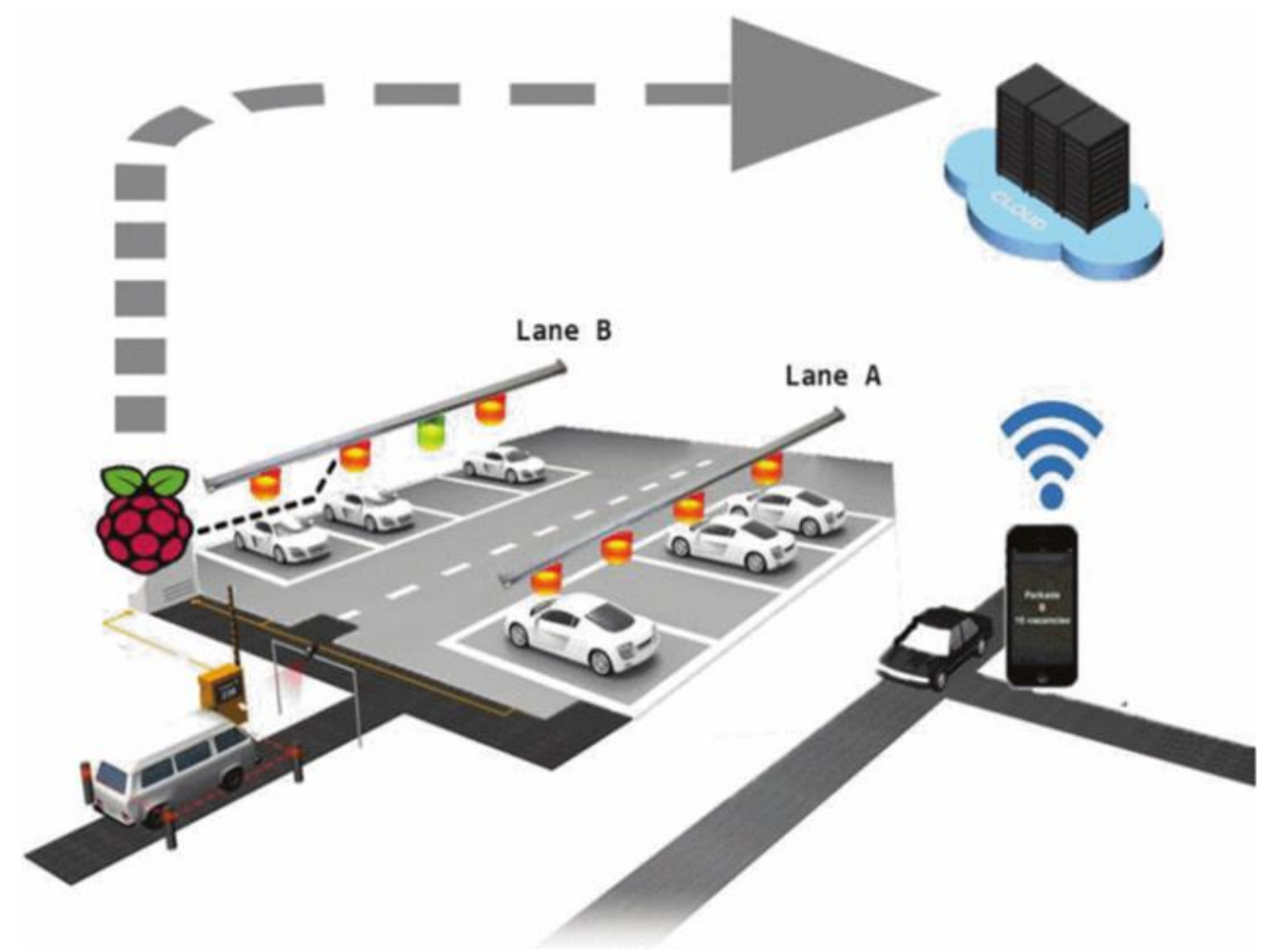

1.2 Figure

\section{PROPOSED SYSTEM}

The parking system is designed in such a way that it is applicable for covered parks, open parks and street side parking. The fig.1 shows the cloud based IOT architecture for smart parking system which contains cloud service provider which provides cloud storage to store information about status of parking slots in a parking area and etc. [10]. The centralized server which manages to store entire smart parking systems information such as number of slots, availability of vehicles etc. And this information will be accessed through some secured gateways through network. This smart parking system which consists of several components. And their functionality includes:

- Centralized server: maintains databases which contain information about parking spaces present in the city.

Proceedings of the $3^{\text {rd }}$ National Conference on Image Processing, Computing, Communication, Networking and Data Analytics (NCICCNDA 2018) 
- Raspberry pi: the microcontroller which is used to implement our parking system and it is attached with raspberry pi camera.

- Image capture: Pi-camera is used to capture the picture of parking area continuously to validate the slots which either filled or empty.

- Navigation system: signals the availability of parking slots to the users and navigates to the exact location of nearest parking area from current location.

- Display device: a monitor or tab is used to display the admin side interface and he is capable of modifying the parking lots by observing the device.

- User device: user can connect with the smart parking system with their smart phones or with some browsers.

The SPS which incorporates the features of raspberry pi 2 and which is attached with pi camera. Where pi camera is mounted on the top of street light lamp posts or at the ceiling of indoor parks. Thus, camera is capable of making survey on each parking slots in parking lots continuously to check whether the particular slot is filled or empty. The fig. 2 presents the structure of smart parking system and it contains some control points on each parking slots which will be used as reference points for the camera. The central server presents information about multiple slots in a single parking area and multiple parking areas in a single city and it is accessed through some protocols such as HTTP, CoAP [12] etc. from any browsers, by which the website is built with basic JSON interface. When the availability of parking slots changes, immediately the information is updated to the central server. Then user can access this stored information using internet International Journal of Scientific and Research Publications, Volume 5, Issue 12, December 2015631 ISSN 2250-3153 www.ijsrp.org from any location. And this information is used by parking operators to determine free parking areas and statistics can be measured at different times in a day on each parking space. The fig. 3 shows the communication between two or more clients and SPS with server. Such that single client can access the information of many parking areas in the city. So by observing the availability of parking slots the user can choose their convenient parking area. Thus particular parking area is navigated from client's current position.

The sufficient user interface is provided to the client so that client can access the clear information about the system. The administrator is capable of creating new parking areas by providing the description or information about the parking area and also manages to add number of parking slots in any particular parking area and even further remove the existing parking slots in a parking area. The updated timing of each parking slot is shown along with unique number. And more importantly this user interface provides the navigation to their destination

\section{CONCLUSIONS}

This designed automatic smart parking system which is simple, economic and provides effective solution to reduce carbon footprints in the atmosphere. It is well managed to access 
and map the status of parking slots from any remote location through web browser. Thus, it reduces the risk of finding the parking slots in any parking area and also it eliminates unnecessary travelling of vehicles across the filled parking slots in a city. So, it reduces time and it is cost effective also.

\section{References}

[1] L. Atzori, A. Iera, and G. Morabito, "The Internet of things: a survey," Computer Networks, vol. 54, no. 15, pp. 2787-2805, 2010.

[2] Kaivan Karimi and Gary Atkinson, - What the Internet of Things (IoT) Needs to Become a Realityll, White Paper, FreeScale and ARM, 2013.

[3] M. Albano, A. Brogi, R. Popescu, M. Diaz, and J. A. Dianes, "Towards secure middleware for embedded peerto-peer systems: Objectives and requirements," in RSPSI '07: Workshop on Requirements and Solutions for Pervasive Software Infrastructures, 2007, pp. 1-6. [Online].

[4] T. Taleb and A. Kunz, "Machine Type Communications in 3GPP Networks: Potential, Challenges, and Solutions," to appear, IEEE Commun. Mag.

[5] http://www.mdpi.com/journal/sensors Sensors 2014, 14, 22372-22393; doi:10.3390/s141222372.

[6] Bilodeau, V.P. Intelligent Parking Technology Adoption. Ph.D. Thesis, University of Southern Queensland: Queensland, Australia, 2010.

[7] Li, T.S.; Ying-Chieh, Y.; Jyun-Da, W.; Ming-Ying, H.; Chih-Yang, C. Multifunctional intelligent autonomous parking controllers for carlike mobile robots. IEEE Trans. Ind. Electron. 2010, 57, 1687-1700.

[8] Faheem1, S.A. Mahmud, G.M. Khan, M. Rahman and H. Zafar,\|A Survey of Intelligent Car Parking Systeml, October 2013.

[9] S. Alam, M. M. R. Chowdhury, and J. Noll, "Senaas: An event-driven sensor virtualization approach for internet of things cloud," in Networked Embedded Systems for Enterprise Applications (NESEA), 2010 IEEE International Conference on, November 2010, pp. 1-6. [Online]. Available: http://dx.doi.org/10.1109/NESEA.2010.5678060

[10] Choeychuen, K. Automatic parking lot mapping for available parking space detection. In Proceedings of the 5th International Conference on Knowledge and Smart Technology (KST), Chonburi, Thailand, 31 January-1 February 2013; pp. 117-121.

[11] Z. Schelby, K. Hartke, and C. Bormann, (Aug. 28, 2013) “Constrained application protocol (CoAP),” CoRE Working Group Internet-Draft. [Online]. 